\title{
Olfaction disorders in patients with pituitary adenoma after endoscopic transsphenoidal surgery: a qualitative study
}

\author{
Xiaomei Zhang ${ }^{1,2 \#}$, Yanqing $\mathrm{Li}^{3 \#}$, Dandan Zhang ${ }^{1,2 \#}$, Fei Huang ${ }^{1,2}$, Yueping Zhong ${ }^{4}$, Xiuqun $\mathrm{Xu}^{2} \wedge$ \\ ${ }^{1}$ Department of Neurosurgery, Affiliated Hospital of Nantong University, Nantong, China; ${ }^{2}$ Department of Nursing, Affiliated Hospital of Nantong \\ University, Nantong, China; ${ }^{3}$ Medical College, Nantong University, Nantong, China; ${ }^{4}$ Department of Surgery, Affiliated Hospital of Nantong \\ University, Nantong, China \\ Contributions: (I) Conception and design: X Zhang, Y Li, D Zhang; (II) Administrative support: Y Zhong, X Xu; (III) Provision of study materials or \\ patients: Y Zhong, X Xu, X Zhang; (IV) Collection and assembly of data: X Zhang, Y Li, F Huang; (V) Data analysis and interpretation: X Zhang, Y \\ Li; (VI) Manuscript writing: All authors; (VII) Final approval of manuscript: All authors. \\ "These authors contributed equally to this work and should be considered as co-first authors. \\ Correspondence to: Xiuqun Xu. Director of Nursing, Department of Nursing, Affiliated Hospital of Nantong University, 20th Xisi Road, Nantong \\ 226001, China. Email: tyxiuqun@126.com; Yueping Zhong. Department of Surgery, Affiliated Hospital of Nantong University, 20th Xisi Road, \\ Nantong 226001, China. Email: 88541265@qq.com.
}

\begin{abstract}
Background: The olfactory function decline of patients with postoperative pituitary adenoma is a common phenomenon. In order to establish a surgical access to the skull base, the upper turbinate, posterior ethmoid chamber and sphenoid sinus need to be operated. We can objectively understand the patient's nasal situation through the scale during the hospital stay, but we know little about the patient's actual feelings and troubles in the home rehabilitation phase.
\end{abstract}

Methods: A qualitative study based on 15 semi-structured face-to-face interviews in a provincial comprehensive hospital was conducted. Participants were purposively selected until data saturation. Each interview was digitally recorded and lasted from 30 to 45 minutes. A trained and experienced interviewer collected all data. The Colaizzi method was used to analyze the data.

Results: A total of six themes were obtained, namely, the patient's perception of symptoms and any accompanying symptoms and signs related to olfactory function decline, negative emotions, decreased appetite, decreased sleep quality, decreased libido, and the need for the continuous care of patients.

Conclusions: A widespread occurrence of impaired smell and nasal function in patients with pituitary adenoma after endoscopic transsphenoidal surgery. It was also noted that nurses should intensify the postoperative nasal care of patients and develop good preventive measures to reduce their postoperative discomfort.

Keywords: Postoperative pituitary adenoma; olfaction disorders; quality of life (QOL); qualitative study

Submitted Oct 11, 2021. Accepted for publication Dec 24, 2021.

doi: 10.21037/apm-21-2920

View this article at: https://dx.doi.org/10.21037/apm-21-2920

\section{Introduction}

A pituitary adenoma is one of the most common central nervous system tumors. Epidemiological studies show that the incidence rate of pituitary adenoma in the general population is $16.7 \%$ (1), with surgery being the primary treatment for pituitary adenomas. Compared with a traditional craniotomy, transsphenoidal minimally invasive surgery for pituitary adenomas has become the first

^ ORCID: Xiaomei Zhang, 0000-0002-6768-7842; Yanqing Li, 0000-0001-7173-2856; Xiuqun Xu, 0000-0001-7384-1116. 
choice as it is less traumatic, has fewer complications, and results in shorter hospital stays for patients (2). The upper turbinate, posterior ethmoid chamber, and sphenoid sinus need to be operated on to establish surgical access to the skull base. With the changes of normal nasal anatomy and inevitable nasal trauma following surgery, many patients will experience olfactory changes (3). Some scholars have reported that the integrity of the nasal cavity in patients undergoing endoscopic surgery had significantly deteriorated 1 month after the operation, and some patients had scabbing, nasal obstruction, nasal allergies, hyposmia, nasal discharge, nasal bleeding, and other undesirable symptoms (4). Previous scholars also evaluated nasal function after endoscopic skull base surgery, and the results showed that nasal respiratory function was at its worst when there was scabbing, and olfactory function was at its worst when there was adhesion (5). Previous studies reported that $19.5 \%$ of patients receiving endoscopic cranial base surgery had nasal fossa adhesions, 39\% had deteriorating olfactory conditions, and $24.3 \%$ had anosmia or hyposmia (6).

A European opinion on the diagnosis and treatment of olfactory disorders confirmed that olfactory dysfunction is associated with reduced integrity and major health problems for sufferers (7). Olfaction plays a key role in the sensory effects of food selection and intake $(8,9)$. Olfactory changes may make patients lose their appetite as olfaction plays a major role in eating behavior. Smell has a great impact on food intake, saliva secretion, and appetite (10). It has been reported that patients with olfactory dysfunction have decreased taste sensitivity, which indicates an interaction between chemical sensory taste, trigeminal nerve function, and olfaction (11).

Furthermore, some studies have reported that patients with olfactory disorders have emotional disorders, such as anxiety and depression $(12,13)$. Higher olfactory performance has also been reported to be associated with positive sexual experiences. Conversely, people with olfactory dysfunction may have a reduced libido $(14,15)$.

At this stage, we can score the perioperative nasal condition of patients using a scale such as the 22-Item Sino-Nasal Outcome Test (SNOT-22) (16), which may help clinicians get an overview of a patient's olfactory or nasal conditions. However, it may be difficult for the data measured by the scale to express disturbances beyond the range of the patient's subjective scale. In general, the experience of olfactory changes in patients after pituitary adenoma surgery is poorly understood. We lack understanding about postoperative nasal discomfort, so patients may not be given effective intervention measures in clinical settings that relieve postoperative nasal discomfort or olfactory dysfunction.

The qualitative study focuses on the meaning, interaction and life experience between people and the research attitude and method of obtaining an overall understanding of the nature of the issue. It also directs how individuals understand and report what they are experiencing. As such, it is the method of choice for exploring a patient's perspective (17). Therefore, this study used phenomenological research methods to conduct indepth interviews relating to olfactory changes experienced by patients after transsphenoidal pituitary adenoma surgery to provide a reference for the development of targeted nursing interventions. We present the following article in accordance with the COREQ reporting checklist (available at https://apm.amegroups.com/article/view/10.21037/apm$21-2920 / \mathrm{rc})$.

\section{Methods}

\section{Study design}

This study chose phenomenological research methods in qualitative research and used semi-structured interviews to collect data. Prior to the interviews, the principal investigators ( $\mathrm{X}$ Zhang and $\mathrm{X} \mathrm{Xu}$ ) prepared a list of topics based on literature and clinical experience. Interview questions were revised iteratively after each interview so that new interesting issues that were raised could be explored in future interviews. Appointments were made with the interviewees the day before the interview, the interview purpose, method, significance, and process were outlined in detail, and the interviewees were informed that the interview materials would be stored anonymously and in code. Emphasis was placed on the principle of voluntary participation, and the informed consent documentation was signed after getting consent from the interviewees, which was kept by both parties. The interviews took place in a quiet, undisturbed conference room. During the interview, the whole process was recorded, and non-verbal behaviors, such as movements, expressions, tone, and other information, were observed and recorded. An interview recording table was used to document the interview content to make up for the shortage of vocal recordings. 


\section{Participants}

We used purposive sampling to enroll patients who had received surgery at the Neurosurgery Department of the Affiliated Hospital of Nantong University from December 2019 to February 2020 and received their first visit to the outpatient department after surgery. The time from the first consultation to the outpatient department was 1 month after surgery. We identified patients who fulfilled the following criteria: (I) over 18 years of age, (II) patients after transsphenoidal pituitary adenoma resection, (III) patients with olfactory disorders according to the SNOT-22 outcome test, (IV) patients who had informed consent and voluntarily participated in the interview and recorded. Patients with other serious physical conditions, mental illness, inability to communicate effectively and nasal experimental interventions from other studies were excluded.

\section{Data collection}

Before implementing this study, the researchers systematically studied the relevant theoretical knowledge of qualitative research and preliminarily mastered the phenomenological research methods, interview methods, and data analysis methods. The researcher himself collected the data. All audio recordings and text data from the study were properly preserved for timely reference during the latter stages of the study. To promote the application of the results of this research to similar patients, the researchers comprehensively retained the language, expressions, movements, and other information from the research subjects when organizing and analyzing the data. Scale measurement was added to the study, which helped assess each patient's olfactory and nasal conditions. Furthermore, the employed scale assisted in confirming that the patients we included suffered from olfactory disorders. One month after their operations, the patients were measured with the SNOT-22, which is a specific scale for nasal diseases.

Patients were interviewed at their preferred locations in the hospital. Main contents: (I) Have you experienced any abnormal perceptions after the operation? What is bothering you most? (II) Could you talk about the sensations of the nasal cavity following the operation? (III) What impact do you think the lack of smell after surgery has had on your life? (IV) How do you deal with these altered perceptions? (V) Do you want to be provided with effective ways to relieve or control symptoms? (VI) What other information do you want to add besides the questions from this interview?

During the interview, interviewers listened carefully, observed, and recorded the patient's movements, expressions, and emotional changes. According to the guidelines of the interview outline and the overall impression of the interviewees, the researchers adjusted the contents and methods of the conversation appropriately, made full use of communication skills such as questioning and silence, encouraged the patients to describe their real impressions without guidance or suggestion, and asked useful questions appropriately. Interviews took 30 to 45 minutes, depending on the amount of information the participant shared. Data saturation criteria included data replication and redundancy when additional interviews failed to uncover new information relating to the study's purpose. After each interview, the recordings were transcribed, and each interviewee was numbered in order from N1 to N15. The interview content was archived independently.

\section{Statistical analysis}

Data was collected in hospitals and analyzed based on Colaizzi's phenomenological methodology. Data analysis was carried out by two researchers who had received qualitative research training (both are Master's students participating in this project), and the analysis results were repeatedly discussed until a consensus was reached. Before analyzing the transcripts, the researchers familiarized themselves with the data. The analysis phase included open coding, the development of categories and themes, and textural and structural descriptions. Separate, independent researchers coded the first two interviews. The codes were then discussed until a consensus was reached. A single researcher coded all other interviews. Subsequently, codes were compared and grouped into broader categories, followed by the development and description of themes. During each step of the analysis process, a meeting took place between the two researchers to reach a consensus. The research team also independently analyzed a subset of transcripts and met to refine the interpretation and validate themes to reduce investigator bias and enhance reflexivity. The interviewer also sent a summary of each interview and a preliminary interpretation of the data to each participant, with the participant was asked to judge whether the summary accurately represented their perspective. The analyses were independently performed by three researchers 
Table 1 Patients' score of the SNOT-22

\begin{tabular}{|c|c|c|c|c|c|c|}
\hline Order & Total points & Nasal symptoms & Cough & Ear and face symptoms & Sleep function symptoms & Psychological problems \\
\hline N2 & 58 & 17 & 1 & 7 & 17 & 16 \\
\hline N3 & 36 & 10 & 1 & 7 & 7 & 11 \\
\hline N4 & 46 & 13 & 2 & 7 & 13 & 11 \\
\hline N6 & 34 & 16 & 0 & 3 & 8 & 7 \\
\hline N7 & 69 & 23 & 1 & 9 & 20 & 16 \\
\hline N8 & 49 & 16 & 2 & 6 & 8 & 17 \\
\hline N9 & 65 & 24 & 1 & 9 & 12 & 19 \\
\hline N12 & 33 & 12 & 0 & 4 & 4 & 13 \\
\hline N13 & 44 & 15 & 2 & 7 & 4 & 16 \\
\hline N14 & 83 & 28 & 1 & 12 & 15 & 27 \\
\hline N15 & 61 & 17 & 0 & 6 & 16 & 22 \\
\hline
\end{tabular}

SNOT-22, 22-Item Sino-Nasal Outcome Test.

using NVivo 12 software. Discrepancies were negotiated within the research team during regular meetings until an agreement was reached. Finally, the results are compared to summarize the theme.

\section{Ethics statement}

The study was conducted in accordance with the Declaration of Helsinki (as revised in 2013). This study was approved by the Ethics Committee of the Affiliated Hospital of Nantong University (No. 2019-K003). Before each interview, an explanation of the purpose and method of the interview and the need for recording during the interview was given to the interviewee. The interviewees were informed about the use of anonymity and coding in the research results and writing papers. The interviewees were informed that the intelligence gained from this study would be strictly confidential, and informed consent was signed after mutual confirmation.

\section{Results}

Fifteen patients were included in the final study. The patient's score for nasal distress was $51.46 \pm 14.12$. This indicates that the patients interviewed were at a moderate level of nasal distress (Table 1). One patient had a postoperative cerebrospinal fluid leak, while the others had no complications. Among them were 9 males and 6 females, aged from 28 to 61 years old (Table 2).

According to the descriptions of the participants and analysis of the data, six categories including disturbance of nasal symptoms, trouble with negative emotions, decreased sleep quality of patients, changes in eating behaviors, effect on the quality of sexual life and required nursing support after discharge (Table 3).

\section{Disturbance of nasal symptoms}

Nasal discomfort is the most common complaint of pituitary adenoma after transsphenoidal surgery. The majority of patients in this study complained of varying degrees of nasal obstruction, runny nose, and other symptoms.

\section{Olfaction disorders}

Most of the patients involved in the investigation had olfactory complaints, with most describing different degrees of olfactory disorder. Some participants described that they could not smell at all, while some participants described 
Table 2 General information of patients

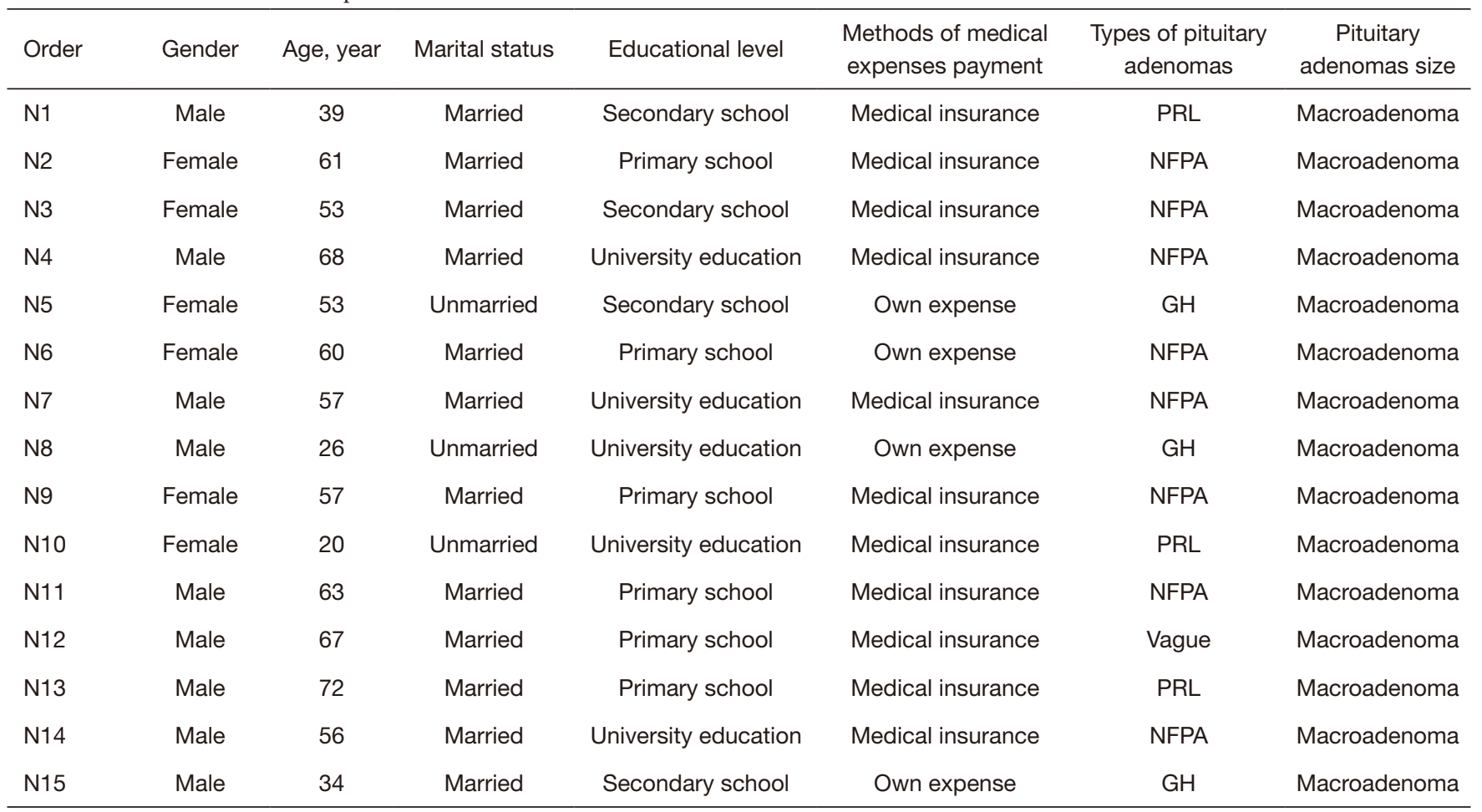

PRL, prolactinoma; NFPA, nonfunctional pituitary adenoma; $\mathrm{GH}$, acromegaly.

Table 3 Superordinate and sub-themes identified in the analysis

\begin{tabular}{|c|c|}
\hline Themes & Sub-themes \\
\hline \multirow{3}{*}{$\begin{array}{l}\text { 1. Disturbance of nasal } \\
\text { symptoms }\end{array}$} & Olfaction disorders \\
\hline & $\begin{array}{l}\text { There are a multitude of nasal } \\
\text { secretions }\end{array}$ \\
\hline & Changes in breathing patterns \\
\hline $\begin{array}{l}\text { 2. The trouble with negative } \\
\text { emotions }\end{array}$ & Anxiety and worry \\
\hline \multirow{2}{*}{$\begin{array}{l}\text { 3. Decreased sleep quality of } \\
\text { patients }\end{array}$} & Trouble falling asleep \\
\hline & $\begin{array}{l}\text { Waking up frequently during } \\
\text { sleep }\end{array}$ \\
\hline 4. Changes in eating behaviors & - \\
\hline 5. Effect on quality of sex life & - \\
\hline \multirow[t]{2}{*}{$\begin{array}{l}\text { 6. Required nursing support } \\
\text { after discharge }\end{array}$} & $\begin{array}{l}\text { Demand for rehabilitation- } \\
\text { related knowledge }\end{array}$ \\
\hline & $\begin{array}{l}\text { The needs for information } \\
\text { support after discharge }\end{array}$ \\
\hline
\end{tabular}

being able only to smell strong odor. Other participants could not smell at all.

Patient 2: "There's a little bit of mucus coming out of my nose occasionally; sometimes it goes through my nose, sometimes it's blocked, but even if it goes through my nose, I still can't smell it."

Patient 15: "I can smell strong scents, like vinegar, durian, but almost nothing else."

Patient 14: "I always experience a strange smell, a little malodorous and bitter. I don't know exactly how to describe it. I smell it whenever I'm awake, but no one else in my family can smell it."

\section{There are a multitude of nasal secretions}

A number of the participants complained of having a smell disorder accompanied by increased nasal secretions. They complained of a strong sensation in the nasal body, and some participants found yellow-green lumpy discharge when pinching the nose slightly, similar to the thick mucus of the common cold. Described were dry patches of 
secretions, some as tiny strips, sometimes accompanied by blood.

Patient 5: "There's a lot of nasal excrement. I dare not pick my nose all the time. I can only roll up a tissue and rub it in my nose to clean it. It's a really unpleasant sensation."

Patient 11: "A few days after the operation, blood wouldn't stop coming from my nose. The situation gradually improved after returning bome, but there is still some blood coming out of my nose."

\section{Changes in breathing patterns}

Some of the participants appeared to suffer from oropharyngeal discomfort, mainly manifested by a dry mouth, heavy breathing, and nausea as a result of switching from passive nasal breathing to breathing orally, and the loss of normal respiratory protection and defense functions combined with nasal secretions blockage and mouth breathing. Some participants complained of breath holding, dyspnea, uneven exhalation between the two nostrils, and extraneous nasal noise while breathing. Other participants complained of dizziness and ear discomfort.

Patient 6: "When I breathe, there is always noise in my nose. Although it is not very loud, people close to me can hear it. I guess that it might be because my nose is not ventilating well."

Patient 12: "One nostril of mine works; the other nostril doesn't. The working nostril always felt warmer than the other. When will it return to normal?"

Patient 2: "I bave a blocked nose, similar to having a cold. My whole nose feels like something is pressing down on it, especially when sleeping. This makes me uncomfortable."

\section{The trouble with negative emotions}

\section{Anxiety and worry}

The appearance or escalation of symptoms can affect a patient's psychological state. Many patients in this study described varying degrees of negative emotions, feeling annoyed, anxious, or worried because of the discomfort of the nasal cavity.

Patient 13: "I worry that my nose won't heal properly. I don't know if this is normal or abnormal. The doctor told me not to bold my breath or cough violently. I was worried that my meninges may have ruptured. I was so scared that I had to hold my breath."

Patient 7: "My nose has been blocked, and there is a lot of nasal mucus. On top of that, I can't smell anything. One day, food in the kitchen was burnt. My family could smell it, but I couldn't. This made me realize that not being able to smell is actually quite dangerous."
Patient 9: "I was at the hospital for only a few days. I did not expect that now, after 1 month, I would still be so uncomfortable. I don't know when I'll be back to normal."

\section{Decreased sleep quality of patients}

\section{Trouble falling asleep}

The degree of nasal ventilation is closely related to sleep quality. During the interviews, most interviewees said that their postoperative sleep quality was poor, and that they believed the main reason for their poor sleep was the discomfort of their nasal cavity. There were also a few participants with concerns for their mental health, worried that they will not fully return to normal life, resulting in insomnia.

Patient 14: "My nose is extremely uncomfortable. It's also uncomfortable bolding my forebead in front of me. I have to turn it over and over for a long time to fall asleep at night."

Patient 13: "I went to bed early, but my nose was bothering me, and I didn't really fall asleep until around midnight."

\section{Waking up frequently during sleep}

In addition, some patients describe frequently interrupted sleep, with some saying that because they are using their mouths to breathe for a long time, the oropharynx becomes dry, resulting in coughing and interrupted sleep. Some mentioned that they are drinking more water because of their dry pharynx, increasing nocturia, causing them to wake during the night.

Patient 12: "It's bard to bave a blocked nose. It's not terrible during the day, but I wake up after a short sleep at night, and I can't get back to sleep properly."

\section{Changes in eating behaviors}

The change in their sense of smell, something which plays a key role in a person's sensory processes of food choice and ingestion, caused participants to lose their appetite. Some participants said they only ate soft foods such as porridge and noodles because of the pain felt when chewing large, hard foods. Other participants had problems with gas exchange and blocked ears while eating, leading to limited swallowing. Some patients experienced nasal postnasal drip, producing anorexia symptoms, which led to significantly reduced food intake and weight loss.

Patient 13: "In the past, when I had a meal, I had a big appetite when I smelled the delicious food. Now it seems that if I can't smell the food, I don't have a good meal." 
Patient 3: "When I chew hard, my nose moves. This can be painful, so I don't dare eat food that needs chewing. This month I basically ate porridge, rotten noodles, drank milk and so on."

Patient 9: "I used to eat a lot. Sometimes I would have a snack if I was hungry at night. Now I eat very little each day, and I estimate that I only eat half as much as before."

\section{Effect on quality of sex life}

When asked if there had been an impact on their sex lives, some patients reported a drop in libido and of having less sex. Some of them said they were more likely to get turned on by a familiar scent in the past. Women were more likely to report a decrease in sexual arousal. Sufferers have been unable to identify the cause of their decreased sexual activity and whether or not their olfactory deterioration is the reason.

Patient 6: "I can't smell the aroma of my wife's body. It seems that there is something missing. It's not easy to get excited."

Patient 11: "I used to bug and kiss my husband. I sensed the masculinity of the smell of smoke and even sweated coming from bim. Now I can't smell it, and that makes me feel uncomfortable."

Patient 7: "There is almost no sex in my life despite being home from surgery for such a long time. It seems that I don't have any desire at all."

\section{Required nursing support after discharge}

\section{Demand for rehabilitation-related knowledge}

Almost all patients indicated that they needed professional guidance after discharge, and only 2 patients had a basic understanding of their condition. Furthermore, 14 patients required professional and personalized disease rehabilitation education from medical staff, and 9 patients needed nasal nursing skills education. Patients wanted to ask a lot of questions, mainly relating to nasal care, observation of nasal secretions, postoperative diet and activities, and so on.

Patient 1: "I didn't stay in the hospital for very long. Before I was discharged from hospital, I forgot to ask specific questions, so I am uncertain about many matters now needing attention. I don't even know if it's normal to feel a little dizzy sometimes."

Patient 13: "I'm too old to remember what the doctor said, and my daughter is away, I don't know what's normal? What's abnormal?"

\section{The needs for information support after discharge}

All patients expected to receive information support from medical staff during their home recovery, and they generally believed that they were prematurely discharged from the hospital soon after surgery and that they had not learned the required skills of home self-care. Many patients were eager to communicate with professionals in a timely fashion when they encountered problems during their home rehabilitation, for example, through social network platforms such as WeChat. They generally believed that the number of nurses' telephone follow-up consultations after discharge was minimal, calling only once or twice, so patients could not comprehend their situation fully and could not receive appropriate guidance.

Patient 15: "I know that they are very busy, and you dare not call the work phone of the department for fear of taking up the clinician's time. But sometimes, when I encounter a problem at home, I really want to find a professional to consult."

Patient 8: "I didn't have the time for a follow-up consult. I caught a cold a few days ago, and something came out of my nose. I didn't know whether it was a normal cold, runny nose or some other reason. I asked, 'Do you have any online consulting platform, such as a WeChat official account? It's not easy for us to come all the way here."

\section{Discussion}

Olfactory nerve receptors are mainly distributed in the upper turbinate of the nasal cavity and the mucosa between the nasal septum. Consequently, any damage or stimulation on the olfactory pathway can cause olfactory dysfunction (18). A previous study investigated the olfactory function of 226 patients with skull base lesions treated by endoscopic surgery 6 months after surgery (19). The results showed that endoscopic surgery could significantly reduce olfactory function. In a study of 81 patients who underwent endoscopic pituitary surgery, significant olfactory changes were noted in $3.9 \%$ of the endoscopic modified transseptal transsphenoidal approach (EMTS-TSA) group and 10.0\% of the endoscopic transsphenoidal approach (ETN-TSA) group (20). These clinical studies have demonstrated that endoscopic transsphenoidal surgery has a noticeable impact on olfactory function. In our study, we found that some patients had impaired olfactory function within 1 month after surgery. Therefore, Surgeons performing the endoscopic pituitary surgery must put in a great deal of effort to preserve the sinonasal function. Due to concerns for decreasing sinonasal quality of life (QOL), modifications to Endoscopic transsphenoidal surgery have 
since been suggested in recent years to potentially decrease nasal morbidity. These have included use of a uninostril approach, preservation of the middle turbinates, avoidance of maxillary antrostomies, and avoidance of raising the nasoseptal flap (4). Therefore, we must diligently monitor our complication rates and continue to innovate surgical techniques to improve surgical remission, reduce sinonasal morbidity and improve patients' overall QOL.

Additionally, some researchers believe that the incidence of olfactory decline is related to postoperative nasal management (21). In recent years, with the application of accelerated rehabilitation surgery, the hospitalization time of patients has shortened, and they are usually discharged 4 to 5 days after surgery. During hospitalization, clinicians often pay more attention to cerebrospinal fluid rhinorrhea, postoperative endocrine disorders, and other serious complications, but less attention to olfactory disorders. Due to the development of modern minimally invasive surgical procedures and the higher requirements of patients for their overall QOL, clinicians need to pay more attention to the postoperative nasal state of patients. In other words, doctors and nurses need to invest more energy in researching effective treatment and care measures.

The interview results of this study demonstrate that patients with pituitary adenomas are disturbed by olfactory changes, accompanied by different degrees of changes in their mood, appetite, sleep, sexual desire, and social interaction. Moreover, these complications will have a greater impact on patient's satisfaction with their operation and subsequent recovery. Therefore, patients should be informed of the possible consequences of surgery on olfaction and the possibility of negative outcomes before the operation to develop their disease-related knowledge and aid their comprehension of the procedure. At the same time, we should strengthen the management of the nasal cavity and evaluate olfaction during the perioperative period, especially continuing to pay attention to the condition of the nasal cavity after hospital discharge. Overall, nurses should use scientifically reliable evaluation tools such as scales and objective olfactory threshold and identification tests that may obtain more reliable quantitative data of olfactory function and make a timely and accurate evaluation of patients' olfactory function, and provide a basis for timely clinical treatment and intervention.

The interview results reflected patients with olfactory dysfunction showed varying degrees of emotional reactions such as anxiety, depression, loss, and worry after surgery, consistent with the relevant research reports $(12,22)$. The rapid development of structural and functional imaging technology reveals the possible reasons: areas of the brain involved in olfactory processing, such as the insula, hippocampus, and thalamus, overlap with areas affecting mood $(23,24)$. As damage to olfactory sensation will lead to the loss of the ability to detect and recognize chemical stimuli in the environment, the odor can be used as a warning signal to remind people of the danger from fire, natural gas, or deteriorated food eliminated. Losing this function will make individuals feel vulnerable, unsafe, and worried. At the same time, on account of the olfactory bulb is the first cortical relay of the olfactory system and directly projecting to the marginal area involved in emotional processing and memory formation, patients with olfactory impairment are more likely to have adverse emotional reactions and negative emotions can further aggravate physical symptoms, including sleep disorders, loss of appetite, loss of sexual desire, social withdrawal, and decreased activity. In this regard, nurses should be more aware of the emotional stability of patients during the perioperative period. To find the actual difficulties and psychological reactions of patients, strengthen communication with patients, dynamically understand the psychological state of patients, and encourage patients to express their worries and reduce psychological pressure are methods employed by nursing staff. In addition, psychological care from family is also particularly important in the stage of family nursing. Doctors and nurses can teach self-regulation and self-control methods of negative emotions, educate family members on communicating and giving emotional support. For some patients, complaining about reducing social activities and inclusion of peer support intervention should be encouraged $(25,26)$. The use of online peer education breaks the limitation of time and geography and provides emotional support and information for users through communication and sharing common experiences, which may help alleviate negative emotions.

In the face of postoperative olfactory changes, most of the respondents did not employ positive coping strategies. They were troubled by their olfactory disorders and accompanying symptoms and were unsure how to manage them. Almost all interviewees expressed the hope of getting professional guidance from doctors and nurses, especially relating to communication with clinicians following hospital discharge. We can increase the number of followup measures, such as internet usage, and the inclusion 
of information technology to overcome difficulties of time or geography (27). Online medical resources that enable follow-up management of remote patients with chronic diseases should be made available so the patients' condition after discharge can be understood and to provide corresponding symptom management strategies $(28,29)$.

Postoperative nasal cavity nursing is an important factor in promoting the healing and rehabilitation of patients. Before discharge, patients should be repeatedly instructed on cleaning the blood clots and secretions in the nasal cavity correctly and promptly to avoid blocking the paranasal sinus opening, obstructing drainage, and potential infection (30). The examination of olfactory function is also highly significant, especially during continued followup after a patient's discharge. Abnormal symptoms might be found in time through continuity of nursing care, and patients can be directed to the hospital for nasal irrigation, nasal medication, and other treatments to recover the physiological function of the nasal cavity and paranasal sinuses and improvement of olfactory function after the operation can be promoted.

In addition to the treatment of the nasal state, simple olfactory training methods to promote the improvement of olfactory function may be attempted. It has been reported that olfactory training with four different scents twice a day can improve olfactory disorder, which may be related to the structural changes of olfactory processing regions in the brain (31). Compared with other treatments, non-invasive and low-cost olfactory training is becoming increasingly recognized because of its safety and curative effects in the last 2 years. At present, olfactory training is rarely reported in China (32). An urgent matter to be addressed is to seek enhancement of the international exchange of information to learn advanced methods and explore more effective intervention measures for olfactory recovery of patients after surgery, and to choose olfactory training methods with good compliance levels that are easy to implement at home, which may improve the olfactory function of patients to the greatest extent during the period of family nursing.

In addition, for most patients complaining of dry mouth, nursing staff can educate patients to use various moist solutions to alleviate the problem $(33,34)$. For instance, patients may make their lemon water to promote saliva secretion or use cucumber slices and honey to keep their mouths moist. In order to relieve dry mouth symptoms during the night, a humidifier and moisturizing lip balm might be used. In order to improve the adaptability and tolerance of patients, adaptive nasal blocking training and breathing training before operating should be attended to. For patients with a loss of appetite, nurses should listen to the patient's concerns and explain the correct diet concepts and the importance of a normal diet. Moreover, the active participation of the patients' family members, educate the family to select the foods they eat every day, and select light and moderate food with meat and vegetables according to the patients' previous taste should be sought.

Regarding patients with decreased sexual desire, an objective and caring attitude to discussing this matter with patients and their spouses should be employed, including open communication channels and eliminating patients' worries about sharing private information. Nurses should explain to patients and their spouses the possible impact of olfactory changes on sexual desire and increase their understanding of the condition and their situation. Educating a patient's spouse to choose the right moment for sexual interaction in their relationship and to give patients pleasure to improve the sexual satisfaction of couples is beneficial (35).

\section{Strengths and limitations}

This study reveals nasal complications experienced by patients, which provides a research basis for olfactory assessment and the management of postoperative patients with pituitary adenoma, and also serves as a theoretical basis for medical personnel to implement targeted interventions, outlining key directions of nursing interventions. However, the study has several limitations. First, the single point of time for data collection must be considered a potential limitation. Second, patient obsession may also be affected by abnormal hormone levels, which we need to pay attention to in future studies. Third, regarding sex life, not every patient has an active sex life, and few patients are willing to talk about this problem at length, with more patients choosing to avoid discussion or only say a little. Therefore, there may be inaccuracies in the representation of patients with sexual distress. In addition, due to memory bias, some patients cannot describe the specific occurrence of a symptom or the period of improvement or aggravation.

\section{Conclusions}

Combined with clinical practice, this study conducted semistructured interviews and phenomenological data analysis 
on 15 patients after transsphenoidal pituitary adenoma surgery. This study suggests a not uncommon occurrence of impaired smell and nasal function in patients with pituitary adenoma after endoscopic transsphenoidal surgery. Postoperative olfactory changes raised serious symptoms in patients, which affected their mood, appetite, sexual desire, nutrition, health, hobbies, and social interaction to varying degrees. Patients were most concerned about when they can fully regain their preoperative condition. The inability to recognize scents and breathing difficulties are major problems, both physical and psychological, which gave patients a sense of insecurity and reduced their QOL. Overall, there is still a long way to improve the state of patient care and explore more effective treatment methods to accelerate the recovery of olfactory function. According to the results of our interviews, doctors should recognize more points of attention during nasal operations, while nurses may also have more specific modes of care that should be considered especially during the period of family nursing.

\section{Acknowledgments}

We would like to extend our sincere gratitude to our patients who were willing to participate in our study, share their stories and experience with us, and help and support people around. Thanks to J. Collie and J. Chapnick for their help with the language of the paper.

Funding: This work was supported for the conduct of the research by Project of Jiangsu Modern Hospital Management Research Center (No. JSY-3-2019-067); Science Foundation of Nantong City Grant (Grant Nos. MS12020011, MSZ18100).

\section{Footnote}

Reporting Checklist: The authors have completed the COREQ reporting checklist. Available at https://apm. amegroups.com/article/view/10.21037/apm-21-2920/rc

Data Sharing Statement: Available at https://apm.amegroups. com/article/view/10.21037/apm-21-2920/dss

Peer Review File: Available at https://apm.amegroups.com/ article/view/10.21037/apm-21-2920/prf

Conflicts of Interest: All authors have completed the
ICMJE uniform disclosure form (available at https://apm. amegroups.com/article/view/10.21037/apm-21-2920/ coif). All authors report that this work was supported for the conduct of the research by Project of Jiangsu Modern Hospital Management Research Center (No. JSY-3-2019067); Science Foundation of Nantong City Grant (Grant Nos. MS12020011, MSZ18100). The authors have no other conflicts of interest to declare.

Ethical Statement: The authors are accountable for all aspects of the work in ensuring that questions related to the accuracy or integrity of any part of the work are appropriately investigated and resolved. The study was conducted in accordance with the Declaration of Helsinki (as revised in 2013). This study was approved by the Ethics Committee of the Affiliated Hospital of Nantong University (No. 2019-K003), and obtained signed informed consent from all patients.

Open Access Statement: This is an Open Access article distributed in accordance with the Creative Commons Attribution-NonCommercial-NoDerivs 4.0 International License (CC BY-NC-ND 4.0), which permits the noncommercial replication and distribution of the article with the strict proviso that no changes or edits are made and the original work is properly cited (including links to both the formal publication through the relevant DOI and the license). See: https://creativecommons.org/licenses/by-nc-nd/4.0/.

\section{References}

1. Inoshita N, Nishioka H. The 2017 WHO classification of pituitary adenoma: overview and comments. Brain Tumor Pathol 2018;35:51-6.

2. Gheorghiu ML, Negreanu F, Fleseriu M. Updates in the Medical Treatment of Pituitary Adenomas. Horm Metab Res 2020;52:8-24.

3. Chaaban MR, Chaudhry AL, Riley KO, et al. Objective assessment of olfaction after transsphenoidal pituitary surgery. Am J Rhinol Allergy 2015;29:365-8.

4. Wu V, Cusimano MD, Lee JM. Extent of surgery in endoscopic transsphenoidal skull base approaches and the effects on sinonasal morbidity. Am J Rhinol Allergy 2018;32:52-6.

5. Garzaro M, Pecorari G, Riva G, et al. Nasal Functions in Three-Dimensional Endoscopic Skull Base Surgery. Ann Otol Rhinol Laryngol 2019;128:208-14. 
6. Dolci RLL, Miyake MM, Tateno DA, et al. Postoperative otorhinolaryngologic complications in transnasal endoscopic surgery to access the skull base. Braz J Otorhinolaryngol 2017;83:349-55.

7. Hummel T, Whitcroft KL, Andrews P, et al. Position paper on olfactory dysfunction. Rhinol Suppl 2017;54:1-30.

8. Proserpio C, de Graaf C, Laureati M, et al. Impact of ambient odors on food intake, saliva production and appetite ratings. Physiol Behav 2017;174:35-41.

9. Janet R, Fournel A, Fouillen M, et al. Cognitive and hormonal regulation of appetite for food presented in the olfactory and visual modalities. Neuroimage 2021;230:117811.

10. Boesveldt S, de Graaf K. The Differential Role of Smell and Taste For Eating Behavior. Perception 2017;46:307-19.

11. Han P, Georgi M, Cuevas M, et al. Decreased electrogustometric taste sensitivity in patients with acquired olfactory dysfunction. Rhinology 2018;56:158-65.

12. Rochet M, El-Hage W, Richa S, et al. Depression, Olfaction, and Quality of Life: A Mutual Relationship. Brain Sci 2018;8:80.

13. Eliyan Y, Wroblewski KE, McClintock MK, et al. Olfactory Dysfunction Predicts the Development of Depression in Older US Adults. Chem Senses 2021;46:bjaa075.

14. Bendas J, Hummel T, Croy I. Olfactory Function Relates to Sexual Experience in Adults. Arch Sex Behav 2018;47:1333-9.

15. Schäfer L, Mehler L, Hähner A, et al. Sexual desire after olfactory loss: Quantitative and qualitative reports of patients with smell disorders. Physiol Behav 2019;201:64-9.

16. Hopkins C, Gillett S, Slack R, et al. Psychometric validity of the 22-item Sinonasal Outcome Test. Clin Otolaryngol 2009;34:447-54.

17. Mays N, Pope C. Qualitative research in health care. Assessing quality in qualitative research. BMJ 2000;320:50-2.

18. Majovsky M, Astl J, Kovar D, et al. Olfactory function in patients after transsphenoidal surgery for pituitary adenomas-a short review. Neurosurg Rev 2019;42:395-401.

19. Kim DH, Hong YK, Jeun SS, et al. Endoscopic Endonasal Transsphenoidal Approach From the Surgeon Point of View. J Craniofac Surg 2017;28:959-62.

20. Hong SD, Nam DH, Kong DS, et al. Endoscopic Modified Transseptal Transsphenoidal Approach for Maximal Preservation of Sinonasal Quality of Life and
Olfaction. World Neurosurg 2016;87:162-9.

21. Postalci L, Erdim I, Demirgil B, et al. Late Postoperative Rhinological Complications After Microscopic Transnasal Hypophysectomy. Turk Neurosurg 2017;27:182-6.

22. Kohli P, Soler ZM, Nguyen SA, et al. The Association Between Olfaction and Depression: A Systematic Review. Chem Senses 2016;41:479-86.

23. Croy I, Hummel T. Olfaction as a marker for depression. J Neurol 2017;264:631-8.

24. Höflich A, Baldinger P, Savli M, et al. Imaging treatment effects in depression. Rev Neurosci 2012;23:227-52.

25. Magasi S, Papadimitriou C. Peer Support Interventions in Physical Medicine and Rehabilitation: A Framework to Advance the Field. Arch Phys Med Rehabil 2021. [Epub ahead of print]. doi: 10.1016/j.apmr.2020.09.400.

26. Mizokami-Stout K, Choi H, Richardson CR, et al. Diabetes Distress and Glycemic Control in Type 2 Diabetes: Mediator and Moderator Analysis of a Peer Support Intervention. JMIR Diabetes 2021;6:e21400.

27. Han Y, Lie RK, Guo R. The Internet Hospital as a Telehealth Model in China: Systematic Search and Content Analysis. J Med Internet Res 2020;22:e17995.

28. Kajiwara K, Kako J, Noto H, et al. The Potential for the Internet and Telehealth in Caregiver Support. Comment on "Using Technology to Facilitate Fidelity Assessments: The Tele-STAR Caregiver Intervention". J Med Internet Res 2021;23:e14953.

29. Malliaras P, Cridland K, Hopmans R, et al. Internet and Telerehabilitation-Delivered Management of Rotator Cuff-Related Shoulder Pain (INTEL Trial): Randomized Controlled Pilot and Feasibility Trial. JMIR Mhealth Uhealth 2020;8:e24311.

30. Tzelnick S, Alkan U, Leshno M, et al. Sinonasal debridement versus no debridement for the postoperative care of patients undergoing endoscopic sinus surgery. Cochrane Database Syst Rev 2018;11:CD011988.

31. Al Aïn S, Poupon D, Hétu S, et al. Smell training improves olfactory function and alters brain structure. Neuroimage 2019;189:45-54.

32. Yan XG, Gao X, Sun ZF, et al. Efficacy and associated factors of olfactory training in the treatment of olfactory dysfunction. Zhonghua Er Bi Yan Hou Tou Jing Wai Ke Za Zhi 2018;53:815-9.

33. Piaton S, Duconseille A, Roger-Leroi V, et al. Could the use of saliva substitutes improve food oral processing in individuals with xerostomia? A systematic review. J Texture Stud 2021;52:278-93. 
34. Ludwar L, Mannel H, Hamacher S, et al. Oil pulling to relieve medication-induced xerostomia: A randomized, single-blind, crossover trial. Oral Dis 2022;28:373-83.

Cite this article as: Zhang $\mathrm{X}$, Li Y, Zhang D, Huang F, Zhong Y, Xu X. Olfaction disorders in patients with pituitary adenoma after endoscopic transsphenoidal surgery: a qualitative study. Ann Palliat Med 2022;11(7):2235-2246. doi: 10.21037/apm21-2920
35. Maleki M, Mardani A, Ghafourifard M, et al. Qualitative exploration of sexual life among breast cancer survivors at reproductive age. BMC Womens Health 2021;21:56. 